\title{
Cells under pressure
}

A tiny silicon chip internalized by cells measures intracellular pressure changes.

Cell biologists are growing to appreciate the importance of mechanical loads, forces and pressures on biological processes such as development or disease progression. Pressures in particular, however, have been difficult to measure directly or without damaging the cell membrane.

Cell mechanical measurements are one area where tools developed using nanotechnology principles appear poised to
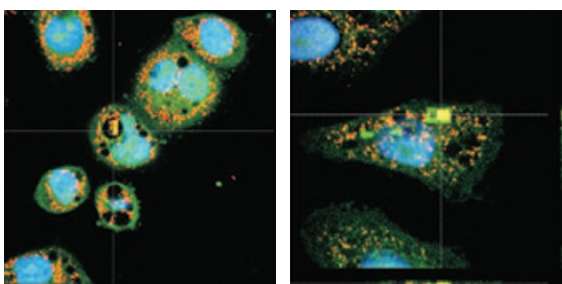

HeLa cells internalize tiny pressure sensor chips. Reprinted from Nature Nanotechnology. make a big impact. To date, though, most nanotechnology devices developed for cell biology applications have only permitted extracellular or invasive types of analyses.

With a new design for an intracellular pressure sensor, José Antonio Plaza's team at the Microelectronic Institute of Barcelona (IMB-CNM) in collaboration with Teresa Suárez's group at the Biological Research Center (CIB) (two Spanish National Research Council research centers) in Spain shrunk a sensor chip down until it was small enough to be internalized by living cells. Semiconductor and microelectronics technology now allows even chips with nanometer-sized structures to be made, which are much smaller than living cells, notes Plaza. "So why not produce chips small enough to be internalized in living cells?" he says.

The $4 \mu \mathrm{m}$ by $6 \mu \mathrm{m}$ pressure sensor chip designed by Plaza's team is based on a FabryPérot resonator, which consists of two silicon-based membranes separated by a vacuum gap. These membranes act as parallel reflecting mirrors; external pressure deflects

\section{SYNTHETIC BIOLOGY}

\section{THE INFLUENCE OF A HOST}

\section{Dissecting the effect of a host's genetic background on circuit performance will} allow better design.

What is taken for granted in many fields of engineering, that a part performs predictably no matter the context, is still a dream for those engineering biological circuits in living cells. But this dream is getting closer to reality.

When a circuit of heterologous proteins is introduced into bacterial cells, three things affect its behavior: the genetic makeup of the inserted DNA, its interaction with the host physiology and the environment the cell is growing in. It is the long-term goal of Adam Arkin at the University of California, Berkeley, to dissect the individual impact of each.

He considers the problem of predicting the effects of genetic context-i.e., how parts, such as promoters or ribosome-binding sites, impact each other's behavior-as almost solved. This is due in part to a pair of Nature Methods papers from earlier this year where he, in collaboration with Drew Endy of Stanford University, presented standardized transcription- and translation-initiation elements that lead to reliable and predictable gene expression in one host in a standardized environment.

"During the review process of these papers, people asked how does [the performance of the circuits] change with the environment, how does it change with different hosts?" says Arkin. "We now took a shot across the bow to see how parts change in different hosts. There is a huge anecdotal literature on which strain to use for high expression, but people did not know exactly why."

In their recent work, the researchers took a simple circuit consisting of three fluorescent proteins-mVenus and mCherry were controlled by the same constitutive promoter and $5^{\prime}$ untranslated region (UTR); mCerulean was regulated by a ribosomal RNA promoter and a unique $5^{\prime}$ UTR - and expressed it in six different strains of Escherichia coli. "By slightly varying the individual parts, but placing the entire system 
the membranes, changing the size of the vacuum gap and modulating the intensity of reflected light. Confocal laser scanning microscopy is used to illuminate the sensors and read out the intensity of the light that is reflected back.

The sensors can be internalized by HeLa cells by liposome transfection. Because of the highly reflective sensor surface, they are easily detected. The chip volume represented just $0.2 \%$ of the total cell volume. Cells containing the sensors divided normally and remained healthy for over a week, and the sensors did not degrade over this time. Some of the sensors remained in vacuoles, which actually worked to the researchers' advantage, as vacuoles insulate the sensor from organelles and cytoskeletal filaments that can induce small forces, and the uniform refractive index of the vacuole environment produces better confocal microscopy images.

The researchers used the sensor chip to monitor pressure changes in response to osmotic shock. Though osmotic shock is predicted to increase the hydrostatic pressure, the sensors did not measure substantial pressure changes. Such pressure changes might be too small to measure with the current sensor design, because HeLa cells are able to adapt when subjected to osmotic shock, avoiding a large increase in intracellular pressure.

Currently, the sensors can detect pressures of a few hundred millibars. "Extracellular mechanical loads can be on this order," notes Plaza, "however, many biological processes will take place with lower pressures." Plaza's team is working to optimize the sensor design, which will increase sensitivity and expand the biological applicability of the these devices.

They are also exploring how they can apply nanotechnology to develop other types of tiny intracellular sensors. "We think that in the near future, intracellular chips will produce unprecedented knowledge in cell biology," Plaza says.

\section{Allison Doerr}

\section{RESEARCH PAPERS}

Gómez-Martinez, R. et al. Silicon chips detect intracellular pressure changes in living cells. Nat. Nanotechnol. 8, $517-521$ (2013).

in genetic variants of the host strain, we asked how expression of these elements was affected by the host background," says Arkin.

In laboratory-adapted strains, his team found that circuit performance is largely determined by generic cellular parameters, such as the availability of ribosomes. But when they looked at the performance in a mutant collection of 88 more strains, in which single genes had been knocked out, the impact of these generic parameters on circuit expression dropped to 35\%. Instead, particular pathways, such as the pentose-phosphate pathway, seemed to exert more influence on how well the proteins were expressed. Some mutants specifically affected only the expression of one of the proteins. "Being able to assess on a genome-wide scale how every piece of the organism interferes with a circuit will be an important part of the design process," says Arkin. "It may have to be done for every host initially, but ultimately we believe principles will emerge."

He is excited to see the increased interest of the National Institute of Standards and Technology to develop standards for synthetic biology as part of a recently launched program for measuring accuracy in the life sciences. "Once you have standards in place, you can populate foundries with data in a computationally accessible framework," he adds. Researchers can then access this knowledge base and pick the parts and host best suited for their goal.

The next step is to look at the influence of the environment. In collaboration with the Joint Genome Institute and Adam Deutschbauer, a senior scientist in his group, Arkin recently established the Functional Encyclopedia of Bacteria and Archea, an effort to use large mutant libraries of natural hosts and screen for growth in hundreds of environments. What remains to be seen is how particular hosts in particular environments affect circuit performance. "And that," says Arkin, "is what we are going to do next."

Nicole Rusk 